\title{
Solubility Of Anthracene in Ternary Cyclohexane + Propanol + 1-Pentanol and Cyclohexane + Butanol + 1-Pentanol Mixtures
}

\author{
Beth A. Martine, Brooke H. Blake-Taylor, and William E. Acree, Jr.* \\ Department of Chemistry, P.O. Box 305070, University of North Texas, Denton, Texas 76203-5070
}

\begin{abstract}
Experimental solubilities are reported for anthracene dissolved in ternary cyclohexane +1 -propanol + 1-pentanol, cyclohexane +2 -propanol + 1-pentanol, cyclohexane + 1-butanol +1 -pentanol, and cyclohexane +2 -butanol + 1-pentanol solvent mixtures at $298.15 \mathrm{~K}$ and atmospheric pressure. For each of the four ternary solvent systems, 19 compositions were studied. Results of these measurements are used to test the predictive ability of the ternary solvent form of the Jouyban-Acree model. Computations showed that the model predicted the observed solubility behavior to within an overall average absolute deviation of about $1.2 \%$.
\end{abstract}

\section{Introduction}

Accurate prediction of phase equilibrium in solid-liquid mixtures is needed in many manufacturing and pharmaceutical applications, especially in purification processes involving recrystallization. Purification by recrystallization generally follows one of two approaches. The first approach involves dissolving the impure solid material in a "good" solvent and then slowly adding a "non-solvent" to precipitate the solute from solution. In the second approach, an appreciable amount of the solid material is dissolved in a hot solution, and upon cooling, purified crystals of the solute form. Solvent selection is important in both cases, and considerable effort has been given in recent years to developing mathematical equations that accurately predict how solute solubility varies with both temperature and solvent composition.

Over the past 20 years, we have reported experimental solubility data for anthracene and pyrene dissolved in numerous binary solvents. As part of our solubility studies, we have developed a simple predictive method for estimating the solubility of crystalline organic compounds in ternary ${ }^{1-4}$

$$
\begin{array}{r}
\ln x_{\mathrm{A}}{ }^{\mathrm{sat}}=x_{\mathrm{B}}{ }^{\mathrm{o}} \ln \left(x_{\mathrm{A}}{ }^{\mathrm{sat}}\right)_{\mathrm{B}}+x_{\mathrm{C}}{ }^{\mathrm{o}} \ln \left(x_{\mathrm{A}}{ }^{\mathrm{sat}}\right)_{\mathrm{C}}+x_{\mathrm{D}}{ }^{\mathrm{o}} \ln \left(x_{\mathrm{A}}{ }^{\mathrm{sat}}\right)_{\mathrm{D}}+ \\
x_{\mathrm{B}}{ }^{\mathrm{o}} x_{\mathrm{C}}{ }^{\mathrm{o}} \sum_{i=0}^{r} S_{\mathrm{BC}, i}\left(x_{\mathrm{B}}{ }^{\mathrm{o}}-x_{\mathrm{C}}\right)^{i}+x_{\mathrm{B}}{ }^{\mathrm{o}} x_{\mathrm{D}}{ }^{\mathrm{o}} \sum_{j=0}^{s} S_{\mathrm{BD}, j}\left(x_{\mathrm{B}}{ }^{\mathrm{o}}-x_{\mathrm{D}}{ }^{\mathrm{o} j}+\right. \\
x_{\mathrm{C}}{ }^{\mathrm{o}} x_{\mathrm{D}}{ }^{\mathrm{o}} \sum_{k=0}^{t} S_{\mathrm{CD}, k}\left(x_{\mathrm{C}}{ }^{\mathrm{o}}-x_{\mathrm{D}}{ }^{\mathrm{o}}\right)^{k}
\end{array}
$$

and higher-order multicomponent solvent mixtures ${ }^{5}$

$$
\begin{aligned}
\ln x_{\mathrm{A}}{ }^{\mathrm{sat}}=\sum_{I} x_{I}^{\mathrm{o}} \ln \left(x_{\mathrm{A}}{ }^{\mathrm{sat}}\right)_{I}+ & \\
& \sum_{I} \sum_{J>I}\left(x_{I}^{\mathrm{o}} x_{J}^{\mathrm{o}} \sum_{i=0}^{r} S_{I J, i}\left(x_{I}^{\mathrm{o}}-x_{J}^{\mathrm{o}}\right)^{i}\right)
\end{aligned}
$$

based on the extended form Combined Nearly Ideal Binary Solvent (NIBS)/Redlich-Kister solution model. Predictions are based on the measured solubility data in all of the contributing sub-binary solvent mixtures. In eqs 1 and $2, x_{\mathrm{I}}^{\mathrm{o}}$ refers to the

\footnotetext{
* To whom correspondence should be addressed. E-mail: acree@unt.edu.
}

initial mole fraction solvent composition of component $i$ calculated as if the solute were not present, and $\left(x_{\mathrm{A}}{ }^{\text {sat }}\right)_{I}$ denotes the measured solute solubility in pure solvent $i$. The various $S_{I J, i}$ parameters can be evaluated with a least-squares regression analysis. Modified versions of eqs 1 and 2 have been developed to include temperature dependence. Currently the most generalized version of the model is referred to as the Jouyban-Acree model (JAM). ${ }^{6,7}$

In the present study, we report the solubility of anthracene in four ternary solvent mixtures containing cyclohexane and 1-pentanol with either 1-propanol, 2-propanol, 1-butanol, or 2-butanol at $298.15 \mathrm{~K}$. These measurements were performed to provide the scientific community with additional solubility data for solutes dissolved in ternary solvent mixtures. The published literature contains experimental solubility data for solutes dissolved in a large number of binary solvent mixtures; however, solubility data for ternary and higher-order multicomponent solvent mixtures are not very abundant. Results of our measurements are used to test the predictive ability of eq 1 .

\section{Experimental Methods}

Anthracene (Aldrich, 99+ \%) was recrystallized several times from 2-propanone to yield a purified sample having a melting point temperature of $T / \mathrm{K}=489$. Cyclohexane (Aldrich, 99.5 $\%$, anhydrous), 1-propanol (Aldrich, 99+ \%, anhydrous), 2-propanol (Aldrich, 99+ \%, anhydrous), 1-butanol (Aldrich, $99+\%$, anhydrous), 2-butanol (Aldrich, $99+\%$, anydrous), and 1-pentanol (Aldrich, $99 \%$ ) were stored over molecular sieves and distilled shortly before use. Gas chromatographic analysis (Hewlett-Packard HP 5890 gas chromatograph equipped with thermal conductivity detection) showed the solvent mole fraction purities to be $99.7 \%$ or better. Binary solvent mixtures were prepared by mass so that composition could be calculated to $0.0001 \mathrm{~mol}$ fraction.

Excess solute and solvent were placed in amber glass bottles and allowed to equilibrate in a constant temperature water bath at $T / \mathrm{K}=298.15 \pm 0.05$ for at least three days (often longer) with periodic shaking to facilitate dissolution of the solid. Attainment of equilibrium was verified by repetitive measurements after a minimum of three additional days and by approaching equilibrium from supersaturation by pre-equilibrat- 
Table 1. Experimental Mole Fraction Solubilities of Anthracene, $x_{\mathrm{A}}{ }^{\text {sat }}$, in Two Ternary Cyclohexane (B) + Propanol (C)

+ 1-Pentanol (D) Solvent Mixtures and Two Ternary Cyclohexane (B) + Butanol (C) + 1-Pentanol (D) Solvent Mixtures at 298.15 K

\begin{tabular}{|c|c|c|c|c|c|}
\hline$x_{\mathrm{B}}{ }^{\mathrm{o}}$ & $x_{\mathrm{C}}^{\mathrm{o}}$ & $x_{\mathrm{A}}{ }^{\text {sat }}$ & $x_{\mathrm{B}}{ }^{\mathrm{o}}$ & $x_{\mathrm{C}}{ }^{\mathrm{o}}$ & $x_{\mathrm{A}}{ }^{\text {sat }}$ \\
\hline \multicolumn{6}{|c|}{ Cyclohexane (B) + 1-Propanol (C) + 1-Pentanol (D) } \\
\hline 0.2935 & 0.4130 & 0.001169 & 0.6331 & 0.2611 & 0.001484 \\
\hline 0.1625 & 0.1919 & 0.001157 & 0.1875 & 0.1483 & 0.001197 \\
\hline 0.1155 & 0.7647 & 0.000828 & 0.1561 & 0.7609 & 0.000863 \\
\hline 0.6479 & 0.2091 & 0.001524 & 0.0988 & 0.4855 & 0.000960 \\
\hline 0.2247 & 0.3263 & 0.001132 & 0.0859 & 0.5946 & 0.000896 \\
\hline 0.4451 & 0.3285 & 0.001327 & 0.3958 & 0.1298 & 0.001381 \\
\hline 0.2065 & 0.5796 & 0.001008 & 0.3232 & 0.5797 & 0.001114 \\
\hline 0.1001 & 0.2660 & 0.001068 & 0.4679 & 0.1395 & 0.001450 \\
\hline 0.6766 & 0.1285 & 0.001597 & 0.4296 & 0.4786 & 0.001251 \\
\hline 0.0917 & 0.7588 & 0.000810 & & & \\
\hline \multicolumn{6}{|c|}{ Cyclohexane (B) + 2-Propanol (C) + 1-Pentanol (D) } \\
\hline 0.2848 & 0.4268 & 0.001073 & 0.6495 & 0.2483 & 0.001502 \\
\hline 0.1425 & 0.1957 & 0.001104 & 0.1918 & 0.1384 & 0.001189 \\
\hline 0.1253 & 0.7544 & 0.000683 & 0.1664 & 0.7497 & 0.000726 \\
\hline 0.6658 & 0.1913 & 0.001521 & & 0.4696 & 0.000880 \\
\hline 0.2271 & 0.3334 & 0.001079 & 0.0959 & 0.5702 & 0.000798 \\
\hline 0.4469 & 0.3246 & 0.001281 & & & 0.001347 \\
\hline 0.2019 & 0.5918 & 0.000891 & 0.3352 & 0.5805 & 0.000999 \\
\hline 0.0950 & 0.2645 & 0.001019 & 0.4857 & 0.1323 & 0.001441 \\
\hline 0.6679 & 0.1390 & 0.001603 & 0.4239 & 0.4851 & 0.001146 \\
\hline 0.0818 & 0.7588 & 0.000652 & & & \\
\hline \multicolumn{6}{|c|}{ Cyclohexane (B) + 1-Butanol (C) + 1-Pentanol (D) } \\
\hline 0.3156 & 0.3683 & 0.001261 & 0.6689 & 0.2301 & 0.001523 \\
\hline 0.1487 & 0.1575 & 0.001183 & 0.2101 & 0.1200 & 0.001252 \\
\hline & 0.7288 & 0.000992 & & & 0.001038 \\
\hline 0.6720 & 0.1721 & 0.001574 & 0.0939 & 0.4331 & 0.001072 \\
\hline 0.2491 & 0.2826 & 0.001231 & 0.0916 & 0.5499 & 0.001021 \\
\hline 0.4729 & 0.2823 & 0.001425 & 0.3954 & 0.1115 & 0.001410 \\
\hline 0.2378 & 0.5382 & 0.001144 & 0.3672 & 0.5394 & 0.001266 \\
\hline 0.1010 & 0.2315 & 0.001132 & 0.4887 & 0.1217 & 0.001504 \\
\hline 0.6710 & 0.1187 & 0.001601 & 0.4621 & 0.4380 & 0.001360 \\
\hline 0.0863 & 0.7246 & 0.000963 & & & \\
\hline \multicolumn{6}{|c|}{ Cyclohexane (B) + 2-Butanol (C) + 1-Pentanol (D) } \\
\hline 0.3165 & 0.3765 & 0.001195 & 0.6740 & 0.2312 & 0.001540 \\
\hline 0.1468 & 0.1786 & 0.001135 & 0.1924 & 0.1212 & 0.001203 \\
\hline 0.1291 & 0.7324 & 0.000836 & 0.1782 & 0.7290 & 0.000885 \\
\hline 0.6818 & 0.1714 & 0.001571 & 0.0935 & 0.4444 & 0.000942 \\
\hline 0.2397 & 0.2759 & 0.001161 & 0.1158 & 0.5243 & 0.000915 \\
\hline 0.4738 & 0.2824 & 0.001388 & 0.3968 & 0.1260 & 0.001360 \\
\hline 0.2268 & 0.5405 & 0.001028 & 0.3737 & 0.5330 & 0.001173 \\
\hline 0.0910 & 0.2397 & 0.001062 & 0.4946 & 0.1183 & 0.001454 \\
\hline 0.6935 & 0.1179 & 0.001607 & 0.4723 & 0.4389 & 0.001301 \\
\hline 0.0954 & 0.7189 & 0.000803 & & & \\
\hline
\end{tabular}

ing the solutions at a slightly higher temperature. Aliquots of saturated anthracene solutions were transferred through a coarse filter into a tared volumetric flask to determine the amount of sample and then diluted quantitatively with methanol for spectrophometric analysis at $356 \mathrm{~nm}$ on a Bausch and Lomb Spectronic 2000. Concentrations of the dilute solutions were determined from a Beer-Lambert law absorbance versus concentration working curve. Molar absorptivities of the nine standard solutions varied systematically with molar concentration and ranged from $\epsilon /\left(\mathrm{L} \cdot \mathrm{mol}^{-1} \cdot \mathrm{cm}^{-1}\right)=7450$ to $\epsilon /\left(\mathrm{L} \cdot \mathrm{mol}^{-1} \cdot \mathrm{cm}^{-1}\right)=7150$ for anthracene compositions ranging from $C /\left(\mathrm{mol} \cdot \mathrm{L}^{-1}\right)=6.75 \cdot 10^{-5}$ to $\mathrm{C} /\left(\mathrm{mol} \cdot \mathrm{L}^{-1}\right)=2.25 \cdot 10^{-4}$. Identical molar absorptivities were obtained for select anthracene standard solutions that contained volume fractions up to $5 \%$ of the neat cyclohexane and alcohol cosolvents. Experimental molar concentrations were converted to mass fraction solubility by multiplying by the molar mass of anthracene, volume(s) of the volumetric flask(s) used, and any dilutions required to place the measured absorbances on the Beer-Lambert law absorbance versus concentration working curve and then dividing by the mass of the saturated solution analyzed. Mole fraction solubili-
Table 2. Combined NIBS/Redlich-Kister Parameters Calculated from Anthracene in the Sub-binary Solvent Systems

\begin{tabular}{|c|c|}
\hline solvent $(I)+$ solvent $(J)$ & $S_{I J}^{a}$ \\
\hline \multirow[t]{3}{*}{ Cyclohexane $(I)+$ 1-Propanol $(J)$} & 1.121 \\
\hline & 0.040 \\
\hline & 0.256 \\
\hline \multirow[t]{3}{*}{ Cyclohexane $(I)+$ 2-Propanol $(J)$} & 1.589 \\
\hline & -0.143 \\
\hline & 0.243 \\
\hline \multirow[t]{3}{*}{ Cyclohexane $(I)+1$-Butanol $(J)$} & 0.741 \\
\hline & 0.345 \\
\hline & 0.223 \\
\hline \multirow[t]{3}{*}{ Cyclohexane $(I)+$ 2-Butanol $(J)$} & 1.260 \\
\hline & 0.206 \\
\hline & 0.000 \\
\hline \multirow[t]{3}{*}{ Cyclohexane $(I)+$ 1-Pentanol $(J)$} & 0.591 \\
\hline & 0.479 \\
\hline & 0.010 \\
\hline \multirow[t]{3}{*}{ 1-Propanol $(I)+$ 1-Pentanol $(J)$} & 0.216 \\
\hline & 0.089 \\
\hline & -0.104 \\
\hline \multirow[t]{3}{*}{ 2-Propanol $(I)+$ 1-Pentanol $(J)$} & 0.445 \\
\hline & 0.111 \\
\hline & 0.033 \\
\hline \multirow[t]{3}{*}{ 1-Butanol $(I)+$ 1-Pentanol $(J)$} & 0.055 \\
\hline & 0.033 \\
\hline & -0.016 \\
\hline \multirow[t]{3}{*}{ 2-Butanol $(I)+$ 1-Pentanol $(J)$} & 0.106 \\
\hline & -0.054 \\
\hline & -0.035 \\
\hline
\end{tabular}

${ }^{a}$ Combined NIBS/Redlich-Kister curve-fit parameters are ordered as $S_{I J, 0}, S_{I J, 1}$, and $S_{I J, 2}$.

Table 3. Summarized Comparison between Observed Anthracene Solubilities in Two Ternary Cyclohexane + Propanol + 1-Pentanol Solvent Mixtures and Two Ternary Cyclohexane + Butanol + 1-Pentanol Solvent Mixtures and Predicted Values Based on Equation 1

\begin{tabular}{cc}
\hline ternary solvent mixture & $\%$ dev. $^{a}$ \\
\hline Cyclohexane (B) + 1-Propanol (C) + 1-Pentanol (D) & 1.17 \\
Cyclohexane (B) + 2-Propanol (C) + 1-Pentanol (D) & 1.10 \\
Cyclohexane (B) + 1-Butanol (C) + 1-Pentanol (D) & 1.25 \\
Cyclohexane (B) + 2-Butanol (C) + 1-Pentanol (D) & 1.29 \\
${ }^{a} \%$ Dev $=(100 / N) \sum\left[\left(x_{\mathrm{A}}{ }^{\text {sat }}\right)^{\text {calcd }}-\left(x_{\mathrm{A}}{ }^{\text {sat }}\right)^{\text {exptl }}\right] /\left(x_{\mathrm{A}}{ }^{\text {sat }}\right)^{\text {exptl. }}$; where $N=$
\end{tabular}
19.

ties were computed from mass fraction solubility using the binary solvent initial mole fraction compositions and molar masses of the solute and both cosolvents. Experimental anthracene solubilities in the four ternary cyclohexane + propanol/ butanol +1 -pentanol mixtures studied are listed in Table 1. Numerical values represent the average of between four and eight independent determinations, with the measured mole fraction solubilities being reproducible to within $\pm 1.5 \%$.

\section{Results and Discussion}

Equation 1 expresses the "excess" logarithmic mole fraction solubility, relative to the simple $x_{\mathrm{B}}{ }^{\mathrm{o}} \ln \left(x_{\mathrm{A}}{ }^{\mathrm{sat}}\right)_{\mathrm{B}}+x_{\mathrm{C}}{ }^{\mathrm{o}} \ln$ $\left(x_{\mathrm{A}}{ }^{\text {sat }}\right)_{\mathrm{C}}+x_{\mathrm{D}}{ }^{\mathrm{o}} \ln \left(x_{\mathrm{A}}{ }^{\text {sat }}\right)_{\mathrm{D}}$ arithmetic average, in terms of the Redlich-Kister equation. Published papers ${ }^{1-3,8,9}$ have reported the calculated $S_{I J}$ parameters dissolved in the nine sub-binary systems, as well as the measured mole fraction solubilities in cyclohexane $\left(x_{\mathrm{A}}{ }^{\text {sat }}=0.001553\right), 1$-propanol $\left(x_{\mathrm{A}}{ }^{\text {sat }}=0.000591\right)$, 2-propanol $\left(x_{\mathrm{A}}{ }^{\text {sat }}=0.000411\right), 1$-butanol $\left(x_{\mathrm{A}}{ }^{\text {sat }}=0.000801\right)$, 2-butanol $\left(x_{\mathrm{A}}{ }^{\text {sat }}=0.000585\right)$, and 1-pentanol $\left(x_{\mathrm{A}}{ }^{\text {sat }}=0.001097\right)$. Numerical values of the $S_{I J}$ parameters have been tabulated in Table 2 for convenience.

The predictive ability of eq 1 is summarized in Table 3 for anthracene dissolved in the four cyclohexane + propanol/butanol 
+1 -pentanol solvent systems. Examination of the numerical entries in Table 3 reveals that eq 1 predicts the solubility of anthracene to within an overall average absolute deviation of $1.2 \%$, which is comparable to the experimental uncertainty of \pm $1.5 \%$. For the four systems studied, eq 1 was found to provide very accurate predictions of the observed solubility behavior. Previous studies have shown that eq 1 predicted the solubility of anthracene in eight ternary alkane + alkane + alcohol solvent systems $^{1,2}$ and in 12 ternary alkane + propanol + butanol $^{3,10,11}$ solvent systems to within an overall average absolute deviation of less than $2 \%$. Results of the present study are in accord with these earlier observations.

\section{Literature Cited}

(1) Deng, T.; Acree, W. E., Jr. Solubility of anthracene in ternary propanol + 2,2,4-trimethylpentane + cyclohexane and butanol + 2,2,4trimethylpentane + cyclohexane solvent mixtures. J. Chem. Eng. Data 1998, 43, 1059-1061.

(2) Deng, T.; Hernández, C. E.; Roy, L. E.; Acree, W. E., Jr. Solubility of anthracene in ternary (propanol + heptane + cyclohexane) and (butanol + heptane + cyclohexane) solvent mixtures. J. Chem. Thermodyn. 1999, 31, 205-210.

(3) Deng, T.; Acree, W. E., Jr. Solubility of anthracene in ternary propanol + butanol + cyclohexane solvent mixtures. J. Chem. Eng. Data 1998, 43, 1062-1064.

(4) Debase, E. M.; Acree, W. E., Jr. Solubility of pyrene in ternary propanol + butanol + cyclohexane solvent mixtures at $299.15 \mathrm{~K}$. J. Chem. Eng. Data 2001, 46, 991-993.
(5) Deng, T.; Horiuchi, S.; De Fina, K. M.; Hernández, C. E.; Acree, W. E., Jr. Solubility of anthracene in multicomponent solvent mixtures containing propanol, butanol and alkanes. J. Chem. Eng. Data 1999, 44, 798-802.

(6) Jouyban, A.; Khoubnasabjafari, M.; Chan, H. K.; Acree, W. E., Jr. Mathematical representation of solubility of amino acids in binary aqueous-organic solvent mixtures at various temperatures using the Jouyban-Acree model. Pharmazie 2006, 61, 1-4.

(7) Jouyban, A.; Acree, W. E., Jr. In silico prediction of drug solubility in water-ethanol mixtures using Jouyban-Acree model. J. Pharm. Pharmaceut. Sci. 2006, 9, 262-269.

(8) Roy, L. E.; Hernández, C. E.; Reddy, G. D.; Sanders, J. T.; Deng, T.; Tuggle, M. B.; Acree, W. E., Jr. Solubility of anthracene in binary alkane +2 -ethyl-1-hexanol and alkane +1 -pentanol solvent mixtures at 298.2 K. J. Chem. Eng. Data 1998, 43, 493-495.

(9) Jouyban, A.; Khoubnasabjafari, M.; Acree, W. E., Jr. Predicting solubility of anthracene in non-aqueous solvent mixtures using a combination of Jouyban-Acree and Abraham Models. Chem. Pharm. Bull. 2006, 54, 1124-1130.

(10) Deng, T.; Childress, S. D.; De Fina, K. M.; Acree, W. E., Jr. Solubility of anthracene in ternary propanol + butanol + heptane solvent mixtures. Chem. Eng. Commun. 1999, 172, 217-224.

(11) Deng, T.; Childress, S. D.; De Fina, K. M.; Tina, L.; Acree, W. E., Jr. Solubility of anthracene in ternary propanol + butanol $+2,2,4-$ trimethylpentane solvent mixtures. J. Chem. Eng. Data 1998, 43, 10651067.

Received for review November 21, 2007. Accepted December 17, 2007.

JE700688Z 